\title{
Dissociating semantic and phonological maintenance using fMRI
}

\author{
GEETA SHIVDE and SHARON L. THOMPSON-SCHILL \\ University of Pennsylvania, Philadelphia, Pennsylvania
}

\begin{abstract}
Functional magnetic resonance imaging (fMRI) distinguished regions of neural activity associated with active maintenance of semantic and phonological information. Subjects saw a single word for $2 \mathrm{sec}$, and following a 10-sec delay, made a judgment about that word. In the semantic task, subjects focused on the meaning of the word and decided whether a second word was synonymous with it. In the phonological task, subjects repeated the word silently and decided whether it shared a vowel sound with a nonsense word. Analyses allowed for isolation of neural activity during the maintenance delay. Semantic maintenance elicited greater activity in bilateral inferior frontal gyrus and left middle temporal gyrus regions of interest (ROI). In contrast, there was greater activity for phonological maintenance in the left superior parietal ROI. These results show a frontal-temporal network involved in actively maintaining the meanings of words, and they indicate that semantic and phonological maintenance processes are dissociable within working memory.
\end{abstract}

At any given moment, we are processing, retrieving, or manipulating information in order to comprehend, reason, and problem solve. Our ability to maintain information actively in order to use it for these cognitive tasks is supported by working memory. The multiple-component model of working memory describes a system that includes a phonological loop that temporarily stores speech-based information through the use of a rehearsal mechanism (Baddeley, 1986). Behavioral studies have supported the idea that the maintenance process within the phonological loop is based on speech sounds, and that it occurs through covert articulation of the information to be kept temporarily active (Baddeley, 1966; Con$\operatorname{rad} \&$ Hull, 1964).

The phonological loop, which has also been referred to as verbal working memory, has also been investigated with the use of neuroimaging. The tasks used in such neuroimaging studies have included the presentation of verbalizable materials such as letters (Awh et al., 1996; D'Esposito et al., 1998; Paulesu, Frith, \& Frackowiak, 1993; Smith, Jonides, \& Koeppe, 1996), words (Fiez et al., 1996), numbers (Petrides, Alivisatos, Meyer, \& Evans, 1993), or nonwords (Jonides, Schumacher, et al., 1998), in which subjects were asked to rehearse a set of multiple items over a delay or dynamically update the items held active over varying delays. Generally, imaging studies done with these kinds of tasks have revealed the

This work was supported by the Searle Scholars program and NIH Grant RO1MH60414 to S.T.S. The authors thank Geoff Aguirre, Laura Barde, Stacey Schaefer, Joseph Kable, and Sarah Thompson for their expert advice and assistance on this project. Correspondence concerning this article should be addressed to G. Shivde, Department of Psychology, 3720 Walnut Street, Philadelphia PA 19104 (e-mail: gshivde@ psych.upenn.edu). activation of frontal regions including Broca's area (Brodmann Area [BA] 44), the dorsolateral prefrontal cortex (BA 46/9), and sometimes the supplementary motor area. Also, phonological rehearsal tasks have usually resulted in inferior (BA 40) or superior (BA 7) parietal activations (but see Fiez, 1997). These activations have been found to be bilateral in some studies of verbal working memory, and with a stronger left lateralization in others (see Smith, Jonides, Marshuetz, \& Koeppe, 1998, for a review).

In addition to outlining the neural circuit associated with tasks involving phonological rehearsal, neuroimaging studies can be theoretically useful for providing converging evidence of the dissociations between working memory processes. For example, Smith et al. (1996) demonstrated that positron emission tomography (PET) activity associated with a verbal working memory task was primarily left lateralized, whereas for a spatial working memory task, it was right lateralized or bilateral. A different dissociation, between maintenance and manipulation of information in the dorsal and ventral prefrontal cortex, respectively, has also been proposed on the basis of reviews of neuroimaging studies of working memory, as well as working memory studies using primates (D'Esposito et al., 1998; Petrides, 1994).

Although the phonological loop has been called "verbal" working memory, it is arguable that for verbal materials such as words, semantic content is as salient as the phonological features. Indeed, for many complex cognitive tasks such as language comprehension, problem solving, and reasoning, the manipulation of semantic information is central. It is logical to assume, then, that this semantic information needs to be actively maintained in working memory in order to keep it accessible for further processing. This kind of active semantic mainte- 
nance has seldom been clearly addressed in previous behavioral and neuroimaging studies of verbal working memory. In fact, many neuroimaging studies have used letter or nonword materials in order to minimize the influence of semantic processing in the phonological rehearsal tasks (e.g., Smith \& Jonides, 1997).

Recently, Shivde and Anderson (2003) have used behavioral methods to demonstrate that maintenance of semantic information can be dissociated from phonological maintenance. In a series of experiments they used a delayed-judgment procedure in which subjects were asked to actively maintain the meaning or the sound of a single abstract word over a delay. At the end of the delay, in the semantic condition, the subjects decided whether a second presented word was a synonym of the word that they were keeping in mind (e.g., BUY and PURCHASE). In the phonological condition, the subjects decided whether a nonsense word shared a vowel sound with the word that they were keeping in mind (e.g., BUY and KINE). In both conditions, the subjects performed a concurrent lexical decision task in which there were embedded probe words semantically related to the item that they were maintaining. The subjects showed a difference in reaction times to the semantically related probe words during the semantic, but not the phonological, maintenance condition. This semantic relatedness effect occurred only during active semantic maintenance, and it demonstrates that semantic and phonological working memory components can be dissociated.

Previous neuropsychological evidence also supports a semantic/phonological dissociation. Patients with distinct areas of cortical damage have shown different profiles of impairment on semantic and phonological shortterm memory tasks (Martin \& Romani, 1994; Martin, Shelton, \& Yaffee, 1994; Romani \& Martin, 1999). The semantic task used by Martin et al. (1994) was a modified item recognition task in which subjects saw several words and, after a delay of $2 \mathrm{sec}$, decided whether a probe word shared a category with any of the previously presented items. In the phonological task, the subjects decided whether the probe word rhymed with any of the items in the memory set. Patient A.B., who had damage to the left prefrontal and adjacent parietal cortex, appeared to have difficulty with semantic maintenance, but was better at phonological tasks (although not normal in comparison with control subjects). Patient E.A. showed deficits on phonological working memory tasks, but appeared to have better semantic maintenance than did A.B. (although worse than controls), and had damage to the left temporal and parietal cortices. This double dissociation of semantic and phonological short-term memory abilities in patients suggests that neural activation patterns in non-brain-damaged populations should also show a dissociation between these two maintenance types.

Following on this work, Crosson et al. (1999) conducted an fMRI experiment in which subjects were asked to maintain multiple words in order to decide whether serially presented probe items matched any of the memory set in semantic category, rhyme, or final three letters. These were called the semantic, phonological, and orthographic conditions, respectively. Activation in these conditions was compared with a control task in which consonant strings were maintained and compared with probe items for matching initial and final letters. In comparison with the control, the semantic condition showed activation of the inferior frontal, premotor, and inferior temporal regions. The phonological condition, when compared with the control, showed activation of more dorsolateral prefrontal cortex as well as premotor areas. However, there were no significant differences in activation when the semantic and phonological conditions were compared directly with one another. This makes it difficult to interpret whether these data actually demonstrate a dissociation between semantic and phonological rehearsal. In addition, since this was a blocked design experiment, activation was integrated across the entire trial, including stimulus presentation, encoding, memory delay, and response. Thus, it is possible that the activations observed may have been the result of the semantic or phonological processing that occurs during encoding, or of comparison of each probe item to the memory set, rather than of the semantic or phonological maintenance processes per se.

The goal of the present study was to directly compare the areas of neural activity associated with active maintenance of the semantic and phonological features of a word over a delay. We used the delayed judgment task employed by Shivde and Anderson (2003), in which subjects maintained the meaning or the sound of a single abstract word during the delay (see Figure 1). The synonym and vowel rhyme judgment tasks at the end of the delay as well as the instructions encouraged subjects to use either a semantic or a phonological maintenance strategy, while at the same time matching memory items across the conditions. In addition, using only one item for maintenance allowed a purer measure of maintenance processes, uncontaminated by ordering and manipulation processes that might be present when multiple items were maintained. Using single words was also prudent because we do not have a precise idea what the capacity limitations of semantic working memory may be. Since this was an event-related fMRI design, it was possible to isolate the sustained neural activity during the delay from the response to initial encoding of the item and the judgment at the end of the delay (Zarahn, Aguirre, \& D'Esposito, 1997). This analysis design allowed us to observe the neural circuitry involved in active semantic maintenance and to examine differences between semantic and phonological maintenance.

\section{METHOD}

\section{Subjects}

Eleven neurologically normal adults from the University of Pennsylvania campus took part in the study. Seven of the participants were male, and 4 were female, with an average age of 23 years (range, 19-29). All subjects reported that they were right-handed 


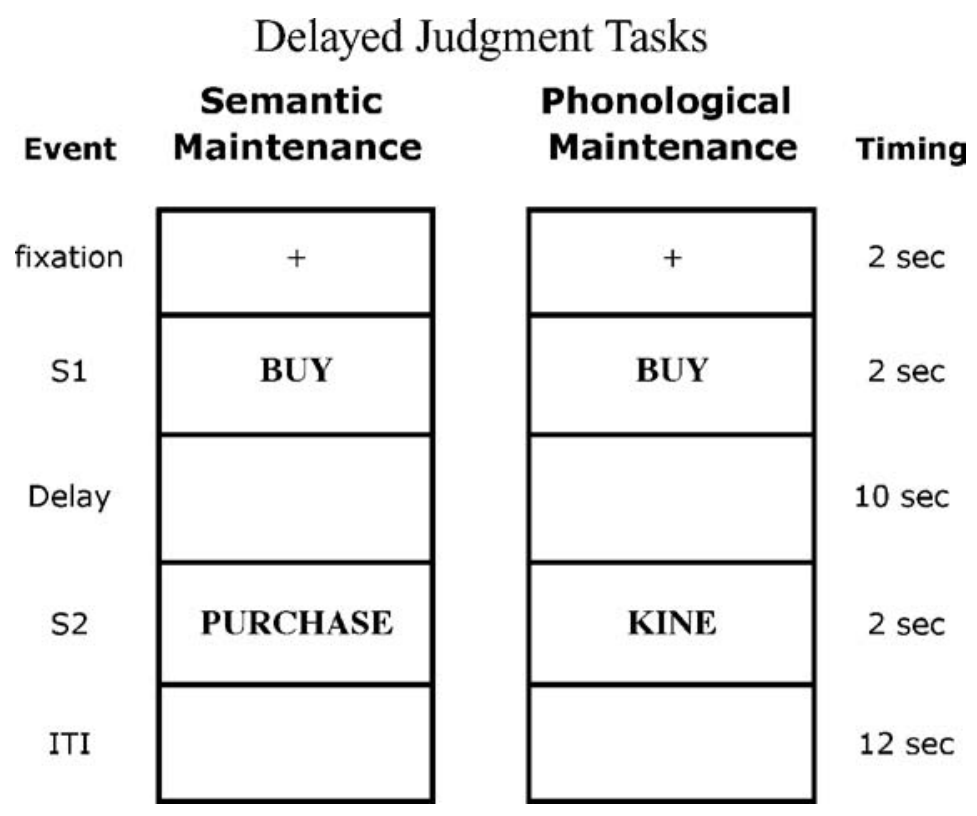

Figure 1. A schematic diagram showing the trial structure of the semantic and phonological maintenance conditions. Timing of the events is indicated on the right.

native English speakers. They also had no reported neurological or psychiatric conditions, and they were not on any psychoactive medications. The subjects were recruited through electronic and paper bulletins on campus and were paid for participation. The subjects gave informed consent and were treated in accordance with institutional human subjects guidelines.

\section{Design and Materials}

All the word stimuli in this experiment were 3-10 letters long and had a word frequency range of 0-399 (median = 147) (Kučera $\&$ Francis, 1967). Seventy words were used as the maintained items (also called S1) for both the semantic and the phonological tasks (see the Appendix). These items had a median concreteness rating of 3.3 on a scale of 1 to 7 , with a range from 1.9 (e.g., freedom) to 5 (e.g., test) (Friendly, Franklin, Hoffman, \& Rubin, 1982). Thirtyfive words were used as target items in the semantic task and were chosen as synonyms of the maintained items (e.g., BUY PURCHASE). The 35 target items (also called S2) for the phonological task were constructed by creating a single-syllable nonsense word that shared a vowel sound with one of the syllables of the maintained word (e.g., BUY KINE). The nonsense word target items were designed to be orthographically dissimilar to the maintained word. In addition, stimuli were constructed for the control task by choosing 70 words and replacing 1 or 2 letters of half of the items in order to create nonwords for the lexical decision task.

Although the data were analyzed as event-related trials, the conditions were blocked so that subjects would not have to switch between phonological and semantic maintenance strategies from trial to trial. The experiment consisted of 14 separate blocks: 7 were semantic, and 7, phonological. These blocks were presented in a random order. Each semantic or phonological block was made up of 10 trials: 5 semantic trials, and 5 control trials randomly intermixed. In total, the subjects completed 35 trials in the semantic condition and 35 trials in the phonological condition. Each block occurred during separate scanning runs that lasted approximately $5 \mathrm{~min}$ each.

\section{Procedure}

Before each block, the experimenter verbally informed the subjects whether they would be doing the semantic or the phonological task. The structure of the trials in the maintenance conditions is depicted in Figure 1.

All trials began with presentation of a fixation cross for $2 \mathrm{sec}$. In the semantic and phonological trials, this was followed by the presentation of a maintained item for $2 \mathrm{sec}$. In the control trials, "WORD/NONWORD" was displayed during this time. Then followed a blank screen during the 10-sec delay. At the end of the delay, the target item was presented for $2 \mathrm{sec}$ in both the semantic and the phonological trials. In the control trials, the letter string for the lexical decision was presented during this time. At this time, subjects indicated their response by a bilateral buttonpress for "yes" or "no." After presentation of the target item, the screen was blank for a 12sec intertrial interval (ITI).

The subjects were instructed that during the delay on the semantic trials, they should keep the word in mind by thinking about the meaning of the word without repeating it to themselves. At the end of the delay, they were presented with another word, they decided whether it had the same meaning as that of the word that they were keeping in mind, and they responded "yes" or "no" with a buttonpress. During the delay on the phonological trials, they were asked to think about the sound of the word by repeating it to themselves. They then saw a single-syllable nonsense word, they decided whether the vowel sound in the target item matched any of the vowel sounds in the word that they were keeping in mind, and they responded with a buttonpress. In both conditions, the subjects were instructed to actively think about the sound or the meaning of the word throughout the entire delay.

The control trials were randomly intermixed with the working memory trials. Each control trial began with the printed signal "WORD/ NONWORD." The subjects were instructed to simply relax during the delay. At the end of the delay, the subjects decided whether the presented letter string was a word or not and responded 
with a buttonpress. The subjects were instructed to rest during the ITI between the trials in each of the conditions.

After the imaging portion of the experiment, the subjects were asked to complete a postexperimental questionnaire about the strategies that they had used during the semantic and phonological delay periods. The entire testing session, including training, testing, and debriefing, lasted $2 \mathrm{~h}$. The subjects were paid $\$ 40$ for their participation.

\section{Imaging Acquisition}

Imaging was performed on a 1.5 T SIGMA scanner (GE Medical Systems) equipped with a fast gradient system for echo planar imaging. A standard radio-frequency (RF) head coil was used, with foam padding to comfortably restrict head motion. High-resolution sagittal and axial T1-weighted images were obtained in every subject prior to the collection of experimental data. We used a gradient echo, echo planar sequence $(\mathrm{TR}=200 \mathrm{msec}, \mathrm{TE}=50 \mathrm{msec})$ to acquire data sensitive to the BOLD signal (Ogawa et al., 1992). Resolution was $3.75 \times 3.75 \mathrm{~mm}$ in plane and $5 \mathrm{~mm}$ between planes, with a total of 21 axial slices acquired per subject. Twenty seconds of gradient and RF pulses preceded the actual data acquisition to allow tissue to reach steady state magnetization. The subjects viewed the stimuli via the Avotec Silent Vision stimulus display system, which consisted of a pair of lightweight fiber-optic glasses mounted on the head coil and a color LCD projector located outside the MRI bore. The stimuli viewed by the subjects were generated by Psyscope 1.1 on a Macintosh, and transmitted to the glasses by fiber-optic image guides.

\section{Image Analysis}

Off-line data processing was performed with programs written in Interactive Data Language (Research Systems, Boulder, CO) and VoxBo software (www.voxbo.org). The data were sinc interpolated in time after image reconstruction and before motion correction. A six-parameter (three translational and three rotational), rigid body least squares realignment routine was performed for motion correction. No subjects had motion that exceeded $2 \mathrm{~mm}$ in any plane. Normalization and spatial smoothing was performed only for the group random effects analyses discussed. All subsequent individual subject region of interest (ROI) analyses were performed on data that were not normalized or spatially smoothed.

A whole-brain group random effects analysis of the main effect of the semantic and phonological conditions was performed in order to choose ROIs for subsequent analyses. The regions of observed activity (at a threshold of $t=2$ ) were in the left and right inferior frontal gyrus (LIFG and RIFG), left middle temporal gyrus (MTG), and left superior parietal gyrus (SPG). For the individual subject ROI data, voxelwise analysis was performed using a general linear model for serially correlated error terms (Worsley \& Friston, 1995). A filter to remove frequencies above $.25 \mathrm{~Hz}$ and below $.02 \mathrm{~Hz}$ allowed us to reduce artifacts in the data. A model for intrinsic serial autocorrelation derived from similarly treated null hypothesis data was assumed (Zarahn et al., 1997). The fixation, maintained item, and target item events were modeled as brief impulses, and the delay was modeled as a 10 -sec boxcar function occurring $4 \mathrm{sec}$ after the start of the trial. These covariates were then smoothed using a standardized estimate of the impulse response function (IRF), to render them appropriate models of the BOLD signal. This method allowed us to discriminate between neural activity associated with the trial component of interest (the delay) and other components. All subsequent analyses focus on the covariate modeling the delay period activity for each of the conditions.

The analyses described below used a functional-anatomical ROI approach, in which activity is averaged across groups of voxels that meet a set of a priori anatomically and functionally def ined criteria. Initially, anatomical ROIs were drawn for each subject, using the sulcal criteria described below. Next, voxels were identif ied within each anatomical ROI that exceeded a threshold for the main effect of maintenance during the delay (in comparison with the ITI activity), collapsed across phonological and semantic trials. Finally, the average fMRI response for the resulting set of voxels in each functional-anatomical ROI was computed separately for the semantic and phonological conditions. These spatially averaged time series served as the dependent variable for all reported analyses.

Anatomical ROIs were drawn on each subject's T1-weighted anatomical images. The inferior frontal gyrus ROI was defined as the gyrus bounded rostrally by the lateral orbital sulcus, caudally by the inferior precentral sulcus, dorsally by the inferior frontal sulcus, and ventrally by the anterior portion of the lateral fissure. The middle temporal ROI was defined as the gyrus extending from the superior to the inferior temporal sulci. The superior parietal ROI was bounded by the parieto-occipital fissure and the intraparietal sulcus.

\section{RESULTS}

\section{Behavioral Results}

Performance on the two working memory conditions was measured in terms of accuracy and response time to decide whether the word they were maintaining was related in meaning to the word at the end of the delay (semantic condition), or shared a vowel sound with it (phonological condition). Neither accuracy $[t(10)=$ $1.52, p=.16]$, nor response time $[t(10)=1.75, p=.11]$ differed between the semantic and phonological tasks. The average accuracy and response times are listed in Table 1.

In a postexperimental questionnaire, subjects rated the extent to which they used a meaning-based or a repetition (subvocal rehearsal) strategy during the semantic and phonological condition blocks. The scale that subjects used was 1 (never) to 5 (always). Figure 2 shows the subject-reported ratings in both conditions. There was an interaction between condition and reported strategy $[F(1,10)=33.0, p<.001]$. Subjects reported using a meaning-based strategy more than repetition during the semantic blocks $[t(10)=3.3, p<.01]$, and repetition more than a meaning strategy during the phonological blocks $[t(10)=5.9, p<.001]$. Thus, subjects seemed to be following instructions in both the semantic and the phonological maintenance conditions.

\section{Imaging Results}

All individual subject analyses were performed on data modeled by the delay period covariate. This covariate models variance uniquely attributable to activity during the delay period, thus excluding possible differences at either stimulus presentation or response. Contrasts

Table 1

Accuracy as Percent Correct and Response Time (in Milliseconds), for Both the Semantic and the Phonological Task Conditions

\begin{tabular}{lccccc}
\hline & \multicolumn{2}{c}{ Accuracy } & & \multicolumn{2}{c}{ Response Time } \\
\cline { 2 - 3 } \cline { 5 - 6 } \multicolumn{1}{c}{ Task } & \% Corr. & $S D$ & & $M$ & $S D$ \\
\hline Semantic & 87 & 5 & & 1,840 & 195 \\
Phonological & 82 & 5 & & 1,963 & 221 \\
\hline
\end{tabular}




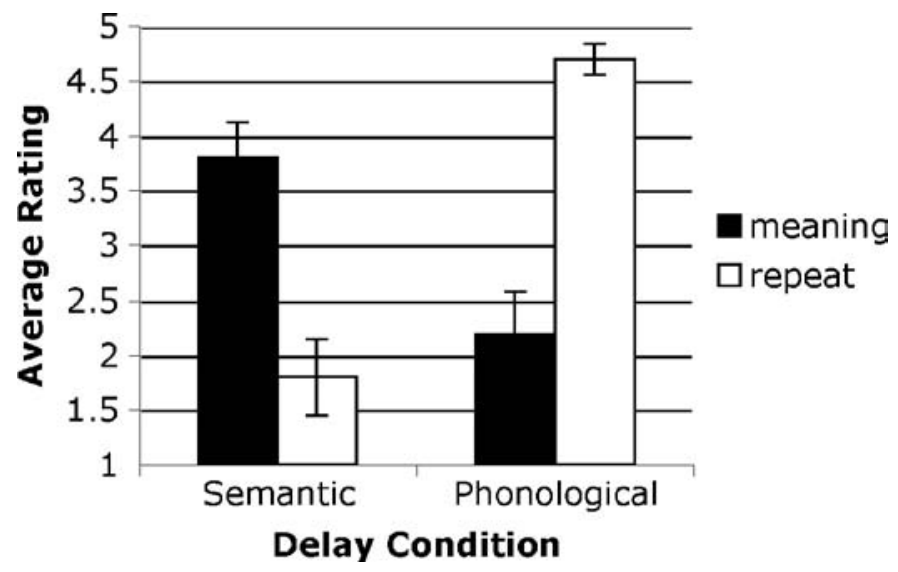

Figure 2. Subjects reported use of repetition and meaning strategy during the semantic and phonological condition blocks.

yielded $t$ statistics with 1714 degrees of freedom, and the false positive rate was controlled at an alpha level of .05 (Bonferoni corrected for the number of voxels per ROI, per subject). Since the main analysis of interest was the direct comparison of the semantic and the phonological delay period activity, the control condition was not included in the following analyses.

For each ROI, voxels were detected that exceeded threshold for the main effect of the maintenance conditions (the sum of the coefficients of the semantic and phonological delay period covariates). Signal in these voxels was then spatially averaged, and contrasts were performed between the semantic and phonological conditions, yielding individual $t$ values. These $t$ values were subsequently used as the dependent variable in a group random effects analysis as an indicator of magnitude of BOLD signal change relative to noise. The mean number of voxels that were spatially averaged for each analysis, and the mean size of each ROI in voxels, is listed in Table 2.

The contrast of semantic to phonological delay period activity showed that there was greater activity during semantic maintenance in both the left $[t(10)=2.5, p<$ $.05]$ and the right $[t(10)=2.3, p<.05]$ IFG regions. Seven of the 11 subjects showed this effect in both the left and the right IFG. There was also greater semantic as opposed to phonological maintenance activity in the left middle temporal gyrus ROI $[t(10)=2.3, p<.05]$, with 9 of the 11 subjects showing the effect. Finally, there was greater phonological as opposed to semantic delay period activity in the left superior parietal ROI $[t(10)=$ $3.9, p<.05$ ], with 7 of 11 subjects showing the effect.

Figure 3 shows the trial-averaged time series data of the percent signal change for the semantic and phonological conditions compared with ITI in each of the ROIs across the delay. As can be seen here, the activity during the semantic delay was greater overall than during the phonological delay bilaterally in the IFG, and in the left middle temporal gyrus. Activity was greater for the phonological delay than for the semantic delay in the left superior parietal gyrus.

An additional post hoc analysis was also performed in order to be certain that the activation differences observed during the delay were not due to residual processing of S1. The data acquired during the first part of the 10-sec delay (Delay 1 ) for each trial were analyzed separately from the data from the second part of the delay (Delay 2). The 10-sec delay period comprised five images, collected every $2 \mathrm{sec}$, so the first two images were coded as Delay 1 and the last three images were coded as Delay 2. The analyses of the Delay 1 and 2 data proceeded exactly as the previous analyses within the four ROIs.

Overall, the split-delay analyses showed that the interactions between the semantic and phonological maintenance conditions were not larger in the first part of the delay than in the second part of the delay. In the LIFG ROI, the direct comparison of semantic and phonological conditions approached significance during Delay 1 $[t(10)=2.12, p=.06]$ and was statistically reliable during Delay $2[t(10)=3.5, p<.01]$. In the RIFG ROI, the comparison between the semantic and phonological conditions again did not reach significance during Delay 1 $[t(10)=1.88, p=.09]$, but did during Delay $2[t(10)=$

Table 2

Mean Number of Voxels (With Range) Activated for the Main Effect of Semantic and Phonological Maintenance as Compared With ITI, and Average Size (in Voxels) of Each Anatomical Region of Interest (ROI)

\begin{tabular}{llrc}
\hline & \multicolumn{2}{c}{ No. Voxels } & \\
\cline { 2 - 3 } Region & $M$ & Range & ROI Size \\
\hline LIFG & 35 & $2-85$ & 224 \\
RIFG & 10 & $2-32$ & 205 \\
LMTG & 12 & $2-31$ & 271 \\
LSTG & 12 & $22-29$ & 151 \\
\hline
\end{tabular}




\section{Region of Interest}

A

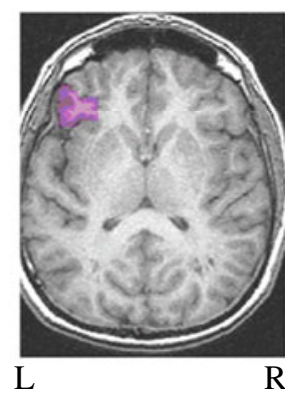

B
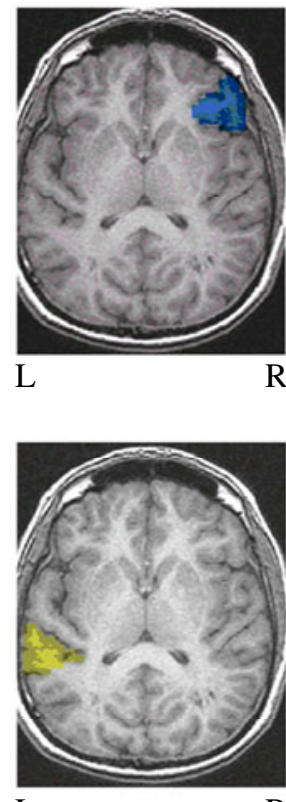

$\mathrm{L}$

$\mathrm{R}$

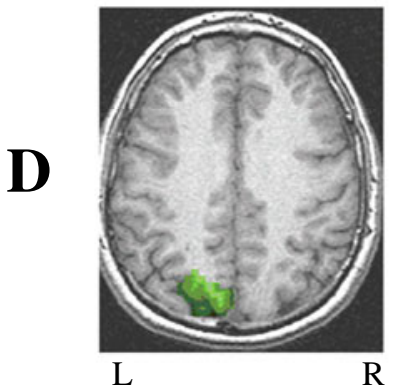

\section{Time Series of Percent Signal Change From S1 to S2}

Left Inferior Frantal Gyrus

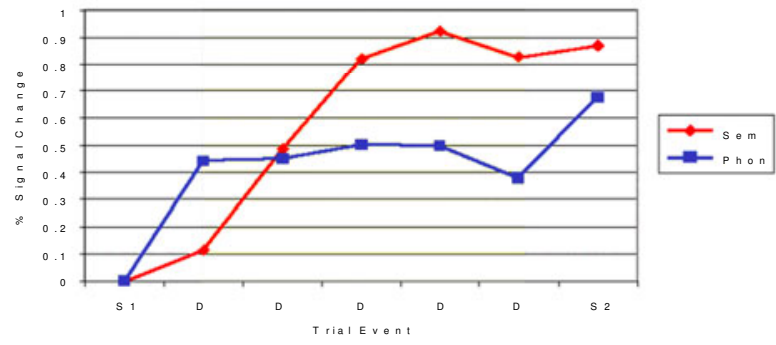

Right Inferiar Frantal Gyrus

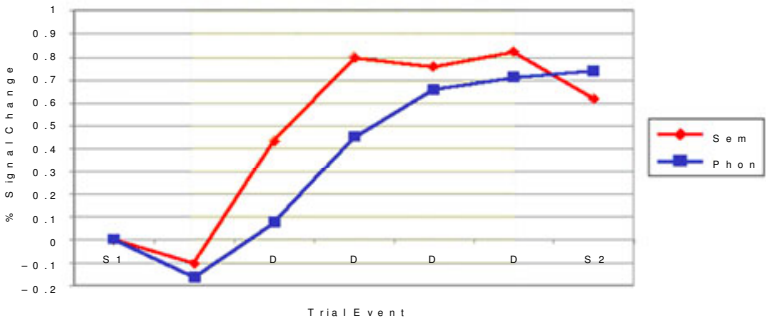

Lefı Middle Temparal Gyrus

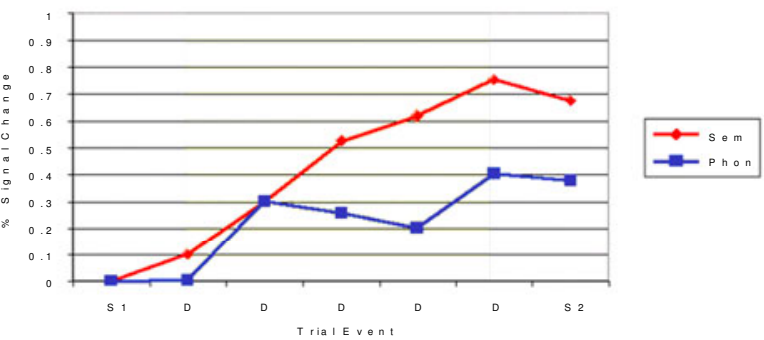

Left Superiar Parietal Gyrus

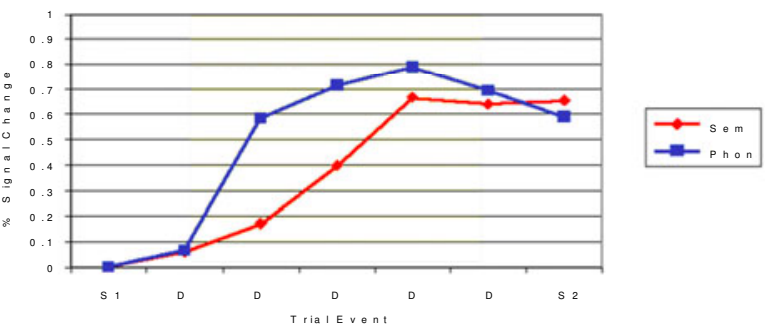

Figure 3. Panels A-D show sample regions of interest (ROIs) from a single subject on the left. Time series data on the right show the percent signal change for the semantic and phonological conditions across the delay in each ROI. S1 on the $x$-axis refers to the maintained item; S2, to the judgment item; and D, to the delay.

$2.31, p<.05]$. Semantic delay activity was also higher than phonological in the MTG at Delay $2[t(10)=2.51$, $p<.05]$, but not at Delay $1[t(10)<1]$. Finally, phonological condition activity was higher than semantic dur- ing both Delay $1[t(10)=2.8, p<.05]$ and Delay 2 $[t(10)=2.39, p<.05]$ in the SPG region. In all of the analyses described above, the effects found during Delay 1 and Delay 2 were not statistically different in 
paired comparisons $[t(10)<1.9$ in all cases $]$. Since none of the effects observed was greater during the first portion of the delay, this analysis further supports the conclusion the activation differences found do not simply reflect encoding processes, but are specific to delay period activity.

\section{DISCUSSION}

Analysis of the delay-related neural activity showed that there were differences when semantic and phonological maintenance were directly contrasted in several anatomical ROIs. In both the left and right inferior frontal gyri, the sustained neural response was greater for semantic than for phonological maintenance. This was also true for the middle temporal gyrus ROI, which may indicate that there is a frontal-temporal circuit involved in active semantic maintenance. The data also show a double dissociation in that the phonological task preferentially activated areas within the superior parietal ROI.

A group random effects analysis of the contrast between semantic and phonological maintenance showed that the peak area of activation in the LIFG was in the anterior extent (MNI coordinates $x=-37, y=22, z=0$ ), and the most dorsal extent of the activation was at MNI coordinates $x=-41, y=30, z=10$. This anterior portion of the LIFG ( BA 45/47) has been shown previously to be involved in tasks requiring semantic processing such as abstract/concrete or living/nonliving category judgments (Demb et al., 1995; Gabrieli et al., 1996; Gold \& Buckner, 2002; Kapur et al., 1994; Poldrack et al., 1999) and semantic monitoring (Petersen, Fox, Posner, Mintun, \& Raichle, 1988). This area can be distinguished from the more dorsal extent of the LIFG (BA 44), which is activated specifically in semantic retrieval (ThompsonSchill, D'Esposito, Aguirre, \& Farah, 1997) and verbal working memory tasks under high selection conditions (Jonides, Smith, Marshuetz, Koeppe, \& Reuter-Lorenz, 1998). Since active semantic maintenance would seem to require refreshing of semantic traces just as phonological rehearsal requires refreshing of phonological traces, it makes sense that an area involved in general semantic processing would be activated during the semantic delay. Previous authors have also made this speculation (Gabrelli et al., 1996; Wagner, Desmond, Demb, Glover, \& Gabrieli, 1997).

In the present experiment, semantic maintenance also led to increased activity in the middle temporal gyrus ROI. Damage to the lateral temporal cortex often results in impairments of semantic memory; for example, a patient may have agnosia that affects only specific categories of objects (Warrrington \& Shallice, 1984). Also, agnosia following left-hemisphere temporal damage can be independent of input or output modality, suggesting damage to the actual semantic representation and not a problem with perceptual or motor recoding of semantic information (Feinberg, Gonzalez-Rothi, \& Heilman, 1986) (for complete review, see Saffran \& Schwartz,
1994). Although not all functional imaging experiments involving semantic processing have shown increased temporal activation (Petersen et al., 1988), several have (Demonet et al., 1992; Klein, Milner, Zatorre, Meyer, \& Evans, 1995; Price, Moore, Humphreys, \& Wise, 1997; Raichle et al., 1994), using tasks such as synonym judgment, semantic monitoring, abstract/concrete judgments, and living/nonliving judgments. In addition, it has been found that the middle temporal gyrus is co-activated with the left inferior prefrontal cortex during controlled semantic retrieval (Gold \& Buckner, 2002). Within the semantic working memory framework, temporal lobe regions may be active during the delay because the semantic features of the item being maintained are repeatedly reactivated by frontal control areas.

In contrast to the semantic maintenance areas described above, the phonological maintenance task showed preferential activity in the left superior parietal ROI (BA 7). Most neuroimaging studies of verbal working memory show increased activity in parietal regions. Usually, the activity occurs in a more inferior region (BA 40) (Smith et al., 1998), but several studies of phonological rehearsal have shown it to occur in the more superior parietal area (Awh et al., 1996; Becker, MacAndrew, \& Fiez, 1999; Davachi, Maril, \& Wagner, 2001). The putative function of the parietal cortex is that it serves as the storage component of verbal working memory maintenance (Awh et al., 1996; Paulesu et al., 1993).

The different patterns of activation in the semantic and phonological conditions indicate a dissociation between these cognitive processes. However, an alternative interpretation is that subjects were using a repetition strategy in the phonological condition and a long-term memory strategy in the semantic condition, thus leading to the different patterns of activation. Specifically, subjects might have used elaborative encoding of S1 and retrieved the information in order to make the synonym judgment of S2. Two pieces of evidence refute this. First, a long-term memory strategy would presumably lead only to a different pattern of activity during the encoding phase of S1 and the judgment phase of S2, but the current analysis isolated the delay-related activity in the semantic condition from the S1 and S2 activity. Second, behavioral studies have shown that when subjects are explicitly asked to use a long-term memory strategy in performing the same delayed semantic judgment task as that described in the present experiment, they do not exhibit the semantic relatedness effect that occurs when they are asked to actively maintain the semantic information. When actively maintaining a word meaning, subjects are slower to respond to a semantically related probe word during the delay. This effect does not occur when subjects simply encode the $\mathrm{S} 1$ item and then retrieve it when presented with the S2 item (Shivde \& Anderson, 2003). Given this evidence, we conclude that the difference in neural activity between the experimental conditions reflects a difference between active semantic and phonological working memory maintenance. 
The present data for the phonological condition do not show activation of specific frontal regions usually found in working memory studies that are assumed to engage the phonological loop. Generally, these regions include Broca's area (BA 44) and sometimes the supplementary motor area (BA 6) (Smith, et al., 1998). One major difference between these studies and the present experiment is that multiple items have almost always been used in previous verbal working memory maintenance tasks. Even the 1-back condition in parametric $n$-back designs requires the processing of more than one item in working memory (Jonides, Schumacher, et al., 1998). It may be that maintaining a single word (well below the presumed verbal span of the average person) is not a strong enough manipulation of the phonological rehearsal process to elicit above threshold activity in frontal regions.

In contrast to that for phonological working memory, the capacity of the putative semantic working memory system is uncertain. Therefore, one word may be closer to the average span for semantic maintenance, which may be why frontal activity was observed in that condition. In this case, a further experiment, using a parametric manipulation of both the phonological and the semantic conditions would be appropriate for determining whether multiple items in the present design would result in detectable frontal activity. Another possibility is that previous studies using multiple-item maintenance or $n$ back updating tasks were actually indexing processes other than phonological maintenance, such as temporal ordering or selection. The present single-item phonological task may be a "purer" measure of the maintenance process.

Martin, Wu, Jackson, Freedman, and Lesch (2003) conducted an event-related fMRI study that did have a load manipulation of semantic and phonological maintenance. The tasks required subjects to retain 1 or 4 words over a 6-sec delay and then compare the word or words with a second word in order to make a synonym or rhyme judgment. The results showed that a left inferior parietal region had greater activity during the phonological task, and that left mid and inferior frontal regions showed a load effect across tasks. However, no region showed greater activity during the semantic task in either a whole-brain or an anatomical ROI analysis. The tasks used in the Martin et al. (2003) study and the present experiment were very similar, but it may be that the use of nonsense words in the present experiment lessened subjects' attention to semantic content during the phonological blocks. Although the results of Martin et al. (2003) did not show that any region responded specifically to the semantic task, it is interesting to note that there was a trend toward a more anterior frontal distribution for the high-load, semantic activity.

Another study that directly contrasted manipulation and maintenance of words and nonwords showed that processing affected activity in the left IFG, but not semantic as opposed to phonological stimulus content (Barde \& Thompson-Schill, 2002). This seems to con- tradict the present finding of a semantic/phonological maintenance contrast within the LIFG. However, there are several important differences between that study and the present one. First, Barde and Thompson-Schill gave subjects five items to maintain (words in the semantic condition, nonwords in the phonological). Also, the semantic task required subjects to order the list of concrete words by size. These factors may have led the subjects to adopt a completely different maintenance strategy than that in the present experiment, in which just one abstract item was maintained for a synonym judgment. In addition, although both experiments involved event-related fMRI, Barde and Thompson-Schill modeled the delay activity as a discrete brief event occurring halfway through the delay, whereas the present analysis used a boxcar to model sustained activity during the delay. Therefore, the two analyses may have captured different aspects of working memory processing. For instance, the present experiment may have been more sensitive to the maintenance processes that were relatively constant over the delay.

In conclusion, the present experiment offers a paradigm that allows for a comparison of semantic and phonological maintenance strategies that is less contaminated by additional executive processing. This comparison shows a clear dissociation of semantic and phonological maintenance in both prefrontal and posterior cortical areas. This provides converging evidence along with the neuropsychological (Martin et al., 1994) and behavioral (Shivde \& Anderson, 2003) studies supporting the idea that semantic maintenance is an independent component of the working memory system.

\section{REFERENCES}

Awh, E., Jonides J., Smith, E. E., Schumacher, E., Koeppe, R. A., \& KATZ, S. (1996). Dissociation of storage and rehearsal in verbal working memory: Evidence from positron emission tomography. Psychological Science, 7, 25-31.

BADDELEY, A. D. (1966). The influence of acoustic and semantic similarity on long-term memory for word sequences. Quarterly Journal of Experimental Psychology, 18, 302-309.

BADDElEy, A. D. (1986). Working memory. Oxford: Oxford University Press, Clarendon Press.

Barde, L. H. F., \& Thompson-Schill, S. L. (2002). Models of functional organization of the lateral prefrontal cortex in verbal working memory: Evidence in favor of the process model. Journal of Cognitive Neuroscience, 14, 1054-1063.

Becker, J. T., MacAndrew, D. K., \& Fiez, J. A. (1999). A comment on the functional localization of the phonological storage subsystem of working memory. Brain \& Cognition, 41, 27-38.

CONRAD, R., \& Hull, A. J. (1964). Information, acoustic confusion and memory span. British Journal of Psychology, 55, 429-432.

Crosson, B., Rao, S. M., Woodley, S. J., Rosen, A. C., Bobholz, J. A., Mayer, A., Cunningham, J. M., Hammeke, T. A., Fuller, S. A., Binder, J. R., Cox, R. W., \& Stein, E. A. (1999). Mapping of semantic, phonological, and orthographic verbal working memory in normal adults with functional magnetic resonance imaging. Neuropsychology, 13, 171-187.

DAVACHI, L., MARIL, A., \& WAgNer, A. D. (2001). When keeping in mind supports later bringing to mind: Neural markers of phonological rehearsal predict subsequent remembering. Journal of Cognitive Neuroscience, 13, 1059-1070. 
Demb, J. B., Desmond, J. E., Wagner, A. D., Vaidya, C. J., Glover, G. H., \& GABRIELI, J. D. (1995). Semantic encoding and retrieval in the left inferior prefrontal cortex: A functional MRI study of task difficulty and process specificity. Journal of Neuroscience, 15, 58705878.

Demonet, J., Chollet, F., Ramsay, S., Cardebat, D., Nespoulous, J., WiSE, R., RASCOL, A., \& FrackowIAK, R. (1992). The anatomy of phonological and semantic processing in normal subjects. Brain, 115, 1753-1768.

D'Esposito, M., Aguirre, G. K., Zarahn, E., Ballard, D., Shin, R. K., \& LEASE, J. (1998). Functional MRI studies of spatial and nonspatial working memory. Cognitive Brain Research, 7, 1-13.

FeinberG, T. E., Gonzalez-Rothi, L. J., \& Heilman, K. M. (1986). Multimodal agnosia after unilateral left hemisphere lesion. Neurology, 36, 864-867.

FIEZ, J. A. (1997). Phonology, semantics, and the role of the left inferior prefrontal cortex. Human Brain Mapping, 5, 79-83.

Fiez, J. A., Raife, E. A., Balota, D. A., Schwarz, J. P., Raichle, M. E., \& Petersen, S. E. (1996). A positron emission tomography study of the short-term maintenance of verbal information. Journal of Neuroscience, 16, 808-822.

Friendly, M., Franklin, P. E., Hoffman, D., \& Rubin, D. C. (1982). The Toronto word pool: Norms for imagery, concreteness, orthographic variables and grammatical usage for 1,080 words. Behavior Research Methods \& Instrumentation, 14, 375-399.

Gabrieli, J. D. E., Desmond, J. E., Demb, J. B., Wagner, A. D., Stone, M. V., Vaidya, C. J., \& Glover, G. H. (1996). Functional magnetic resonance imaging of semantic memory processes in the frontal lobes. Psychological Science, 7, 278-283.

GOLD, B. T., \& BUCKNER, R. L. (2002). Common prefrontal regions coactivate with dissociable posterior regions during controlled semantic and phonological tasks. Neuron, 35, 803-812.

Jonides, J., Schumacher, E. H., Smith, E. E., Koe Ppe, R. A., Awh, E., Reuter-Lorenz, P. A., Marshuetz, C., \& Willis, C. R. (1998). The role of parietal cortex in verbal working memory. Journal of Neuroscience, 18, 5026-5034.

Jonides, J., Smith, E. E., Marshuetz, C., Koe pPe, R. A., \& ReuterLORENZ, P. A. (1998). Inhibition in verbal working memory revealed by brain activation. Proceedings of the National Academy of Sciences, 95, 8410-8413.

Kapur, S., Rose, R., Liddle, P. F., Zipursky, R. B., Brown, G. M., Stuss, D., Houle S., \& Tulving, E. (1994). The role of the left prefrontal cortex in verbal processing: Semantic processing or willed action? NeuroReport, 5, 2193-2196.

Klein, D., Milner, B., Zatorre, R. I., Meyer, E., \& Evans, A. C. (1995). The neural substrates underlying word generation: A bilingual functional imaging study. Proceedings of the National Academy of Sciences, 92, 2899-2903.

KUČERA, H., \& FRANCIS, W. (1967). Computationalanalysis of presentday American English. Providence, RI: Brown University Press.

MARTIN, R.C., \& ROMANI, C. (1994). Verbal working memory and sentence comprehension: A multiple-components view. Neuropsychology, 8, 506-523.

Martin, R. C., Shelton, J. R., \& YAFFEe, L. S. (1994). Language processing and working memory: Neuropsychological evidence for separate phonological and semantic capacities. Journal of Memory \& Language, 33, 83-111.

Martin, R. C., Wu, D., Jackson, D., Freedman, M., \& Lesch, M. (2003). An event-related fMRI investigation of phonological vs. semantic short-term memory. Journal of Neurolinguistics, 16, 341-360.
Ogawa, S., Tank, D. W., Menon, R., Ellermann, J. M., Kim, S. G., MERKLE, H., \& UGURBIL, K. (1992). Intrinsic signal changes accompanying sensory stimulation: Functional brain mapping with magnetic resonance imaging. Proceedings of the National Academy of Science, 89, 5951-5955.

Paulesu, E., Frith, C. D., \& Frackowiak, R. S. (1993). The neural correlates of the verbal component of working memory. Nature, 362, 342-345.

Petersen, S. E., Fox, P. T., Posner, M. I., Mintun, M., \& Raichle, M. E. (1988). Positron emission tomographic studies of the cortical anatomy of single-word processing. Nature, 331, 585-589.

Petrides, M. (1994). Frontal lobes and behaviour. Current Opinion in Neurobiology, 4, 207-211.

Petrides, M., Alivisatos, B., Meyer, E., \& Evans, A. C. (1993). Functional activation of the human frontal cortex during the performance of verbal working memory tasks. Proceedings of the National Academy of Sciences, 90, 878-882.

Poldrack, R. A., Wagner, A. D., Prull, M. W., Desmond, J. E., Glover, G. H., \& GABrieli, J. D. (1999). Functional specialization for semantic and phonological processing in the left inferior prefrontal cortex. NeuroImage, 10, 15-35.

Price, C. J., Moore, C. J, Humphreys, G. W., \& Wise, R. J. S. (1997). Segregating semantic from phonological processes during reading. Journal of Cognitive Neuroscience, 9, 727-733.

Raichle, M. E., Fie Z, J. A., Videen, T. O., Macleod, A. K., Pardo, J. V., Fox, P. T., \& Petersen, S. E. (1994). Practice-related changes in human brain functional anatomy during nonmotor learning. Cerebral Cortex, 4, 8-26.

RoMANi, C. \& MARTIN, R. A. (1999). A deficit in the short-term retention of lexical-semantic information: Forgetting words but remembering a story. Journal of Experimental Psychology: General, 128, 56-77.

SAFFrAn, E. M., \& SCHWARTZ, M. F. (1994). Impairment of sentence comprehension. Philosophical Transactions of the Royal Society of London: Series B, 346, 47-53.

SHIVDE, G., \& ANDERSON, M. C. (2003). On the existence of a working memory system for the active maintenance of semantics. Manuscript submitted for publication.

SMith, E. E., \& Jonides, J. (1997). Working memory: A view from neuroimaging. Cognitive Psychology, 33, 5-42.

SMith, E. E., JonideS, J., \& KoE PPE, R. A. (1996). Dissociating verbal and spatial working memory using PET. Cerebral Cortex, 6, 11-20.

Smith, E. E., Jonides, J., Marshuetz, C., \& Koeppe, R. A. (1998). Components of verbal working memory: Evidence from neur imaging. Proceedings of the National Academy of Sciences, 95, 876882.

Thompson-Schill, S. L., D’Esposito, M., Aguirre, G. K., \& FArAh, M. J. (1997). Role of left prefrontal cortex in retrieval of semantic knowledge: A reevaluation. Proceedings of the National Academy of Sciences, 94, 14792-14797.

Wagner, A. D., Desmond, J. E., Demb, J. B., Glover, G. H., \& GABRIELI, J. D. E. (1997). Semantic repetition priming for verbal and pictorial knowledge: A functional MRI study of left inferior prefrontal cortex. Journal of Cognitive Neuroscience, 9, 714-726.

WARrRington, E. K., \& Shallice, T. (1984). Category specific semantic impairments. Brain, 107, 829-854.

WORSLEY, K. J., \& FRISTON, K. J. (1995). Analysis of fMRI time-series revisited-again. NeuroImage, 2, 173-181.

Zarahn, E., Aguirre, G., \& D Esposito, M. (1997). A trial-based experimental design for fMRI. NeuroImage, 6, 122-138. 
APPENDIX

List of Stimuli

\begin{tabular}{|c|c|c|c|c|c|}
\hline $\begin{array}{l}\text { Maintained } \\
\text { Item }\end{array}$ & $\begin{array}{c}\text { Semantic } \\
\text { Target }\end{array}$ & $\begin{array}{c}\text { Phonological } \\
\text { Target }\end{array}$ & $\begin{array}{l}\text { Maintained } \\
\text { Item }\end{array}$ & $\begin{array}{c}\text { Semantic } \\
\text { Target }\end{array}$ & $\begin{array}{c}\text { Phonological } \\
\text { Target }\end{array}$ \\
\hline afraid & scared & kade & mankind & humanity & jine \\
\hline bad & evil & zak & mistake & error & riss \\
\hline brag & boast & yag & nothing & zero & pring \\
\hline build & construction & pilg & old & aged & yole \\
\hline busy & occupied & pizz & outcome & result & nowt \\
\hline change & alter & tane & part & piece & lah \\
\hline cold & chilly & fole & permit & allow & rit \\
\hline courage & bravery & der & pity & sympathy & zee \\
\hline dead & lifeless & vek & prank & joke & nank \\
\hline different & contrary & zin & purchase & buy & wess \\
\hline disgrace & shame & fiss & quarrel & spat & taw \\
\hline display & exhibit & fape & rascal & scoundrel & jad \\
\hline easy & simple & nee & reject & renounce & bek \\
\hline embrace & hug & tase & rise & ascend & nipe \\
\hline emotion & feeling & po & same & alike & habe \\
\hline endure & survive & byur & shape & form & wabe \\
\hline enjoy & like & leb & skill & talent & vin \\
\hline equal & even & deet & sleep & rest & tees \\
\hline exit & leave & vip & sob & weep & kob \\
\hline fever & temperature & teeb & source & origin & yore \\
\hline find & locate & wibe & speak & talk & veek \\
\hline first & primary & terr & stamina & energy & jad \\
\hline fraud & deceit & kaw & stop & halt & opp \\
\hline freedom & liberty & klebe & strong & muscular & quong \\
\hline glad & happy & kaz & succeed & achieve & wuck \\
\hline happen & occur & dat & tale & story & dail \\
\hline hate & disdain & paze & test & exam & pess \\
\hline help & aid & pelf & think & ponder & grink \\
\hline hike & trek & yibe & tired & weary & sile \\
\hline honest & truthful & dest & tour & expedition & food \\
\hline honor & glory & dahn & triumph & victory & bly \\
\hline hurt & pain & ler & walk & stroll & baw \\
\hline idea & thought & gee & wander & roam & ver \\
\hline imposter & pretender & hin & want & desire & mont \\
\hline long & lengthy & ponk & weak & frail & feeb \\
\hline
\end{tabular}

(Manuscript received November 12, 2003;

revision accepted for publication March 4, 2004.) 